\title{
Chaotic Characteristics and the Application of SVM in the Tool Wear State Recognition
}

\author{
Guan Shan ${ }^{\text {a }}$, Pang Hongyang and Kang Zhenxing \\ Northeast Dianli University school of mechanical engineering, Jilin, China, 132012
}

\begin{abstract}
Metal cutting process is a nonlinear system to obtain the tool wear state and chaos theory are introduced tool wear and feature extraction of acoustic emission signal analysis and classification of tool wear state and wear prediction based on support vector machine (SVM). First, optimal embedding dimension of the time delay of phase space reconstruction of nonlinear dynamic system, the chaotic attractor; secondly, three characteristics: correlation dimension, the largest Lyapunov exponent and the Kolmogorov is extracted from the AE signal denoising feature vector and construct the different wear conditions. Finally, the feature vector is fed into the support vector machine (SVM), and the tool wear condition is classified. Research shows that: the cutting tool wear acoustic emission signal possesses the characteristics of chaos, chaotic characteristic parameters and tool wear status has intrinsic relationship; combined with chaos theory and support vector machine (SVM), can be very good to achieve the tool wear state recognition and prediction.
\end{abstract}

\section{Introduction}

As for automated processing, monitoring the tool wear state can give system error level in real-time, realize cutting compensation automatically, reduce the processing cost effectively and reduce scrap rate [1]. Li Xiaoli [2] used the wavelet analysis method to extract the vibration and acoustic emission tool wear characteristics, set up the neural network model based on fuzzy reasoning and better recognition results were obtained. Tae Jo $\mathrm{KO}$ et al [3] used time series AR model to extract characteristics of cutting force, classify and cluster analysis, and monitor single crystal diamond tool wear state based on the fuzzy recognition technology. Wafaa Rmili et al [4] used a three-axis accelerometer to measure vibration signal during the process of turning, to extract the feature parameters reflect the vibration response, and input the feature parameters to the automatic monitoring system to realize online monitoring of tool wear. The process of tool wear can produce abundant acoustic emission signal. Through tool wear acoustic emission signal sampling, this paper introduced chaos theory analysis, extracted three chaotic characteristic parameters: the correlation dimension and Lyapunov index and Kolmogorov entropy [5], constructed feature vector under different wear conditions, and the characteristic vector were put into the support vector machine (SVM) to identify the tool wear state and forecast tool wear loss.

\footnotetext{
${ }^{\mathrm{a}}$ Corresponding author : guanshan1970@163.com
} 


\section{Test data collection}

Tool wear is nonlinear, and the result of many factors, so in this paper, by using the principle of the orthogonal experiment to simplify the factor levels, extract the key factors (cutting speed v, feeding f, by turning the ap) to do experiment. Test was carried out in CKA6136i CNC lathe, using Kennametal KC9125 carbide coating blade, cutting material is GH625 nickel-based superalloy. Acoustic emission sensor is the NI Company R15, preamplifier is PXPA II broadband acoustic emission amplifier, 1.5 $\sim 2$ MHZ bandwidth, $40 \mathrm{db}$ gain selection, acquisition card is USB6366 NI Company, and sampling rate is $2 \mathrm{MHZ}$.

In order to eliminate the installation error caused by tool change and measuring tool wear loss, when completing a specified cutting time, a cutting edge is no longer used. Take a new knife to cut, after cutting $10 \mathrm{~s}$, parking, record the $6 \sim 10 \mathrm{~s}$ of data, and tool microscope is used to measure the flank wear loss VB. Replace a new tool, cutting $20 \mathrm{~s}$ in the case of cutting condition unchanged. Two pieces of data were recorded, a period of $6 \sim 10 \mathrm{~s}$, a period of $15 \sim 20 \mathrm{~s}$. In theory, the first piece of data and the $6 \sim 10 \mathrm{~s}$ of cutting records for the first time signal, their degree of tool wear is the same. Measure the tool wear after parking. A new knife is used for cutting for a longer time repeatedly, until the cutter grinding is blunt. To illustrate the results of this article, with the cutting speed of $560 \mathrm{r} / \mathrm{min}$, feed speed of $0.3 \mathrm{~mm} / \mathrm{r}$, cutting depth of $0.4 \mathrm{~mm}$, the acquisition of the acoustic emission signal, for example, is described. Figure 1 is the timing signals whose tool wear loss are: $0.10 \mathrm{~mm}, 0.13 \mathrm{~mm}$, $0.16 \mathrm{~mm}$ and $0.23 \mathrm{~mm}, 0.27 \mathrm{~mm}$ and $0.30 \mathrm{~mm}$.
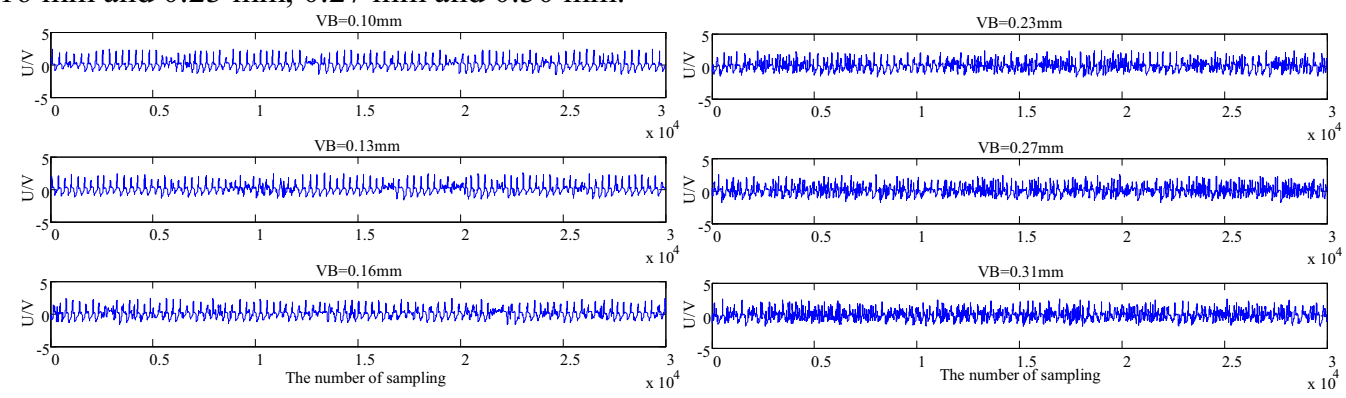

Figure 1. Timing signals of wear loss

\section{Feature extraction based on chaos theory}

The chaos [6] was produced by a deterministic nonlinear dynamic system, it seems to be no rules, similar to the random phenomenon. For nonlinear dynamic system, to fully excavate internal information, we need to expand one-dimensional temporal signal dimension, reconstruct the phase space of dynamic system, and then extract the characteristic parameters of correlation dimension and Lyapunov index and Kolmogorov entropy, which are used for describing system.

\subsection{The phase space reconstruction}

Phase space reconstruction refers to the fact that actual system in the given time series is extended to 3 $\mathrm{d}$ or more higher dimensional space, the key lies in the determination of delay time and embedding dimension. This paper mainly used the autocorrelation method to evaluate delay time $\tau$, CAO method for embedding dimension.

(1)The determination of delay time

Autocorrelation method is to determine the time delay, it is very mature, the basic idea is to solve the linear correlation between the time series. For a given a time series, write the related function, with the increase of time, when autocorrelation function descends to the initial value of 1 to $1 / \mathrm{e}$, the best time delay is to reconstruct phase space, it is best delay time $\tau$ to reconstruct phase space. The selection of different wear stages delay time is shown in figure 2. It can be seen from the diagram, $\tau$ values showed a trend of overall decrease with the increase intent of tool wear. With the increase of tool wear, 
acoustic emission phenomenon grows with the increase of friction between flank and artifacts, the increase number of ringing per unit time makes maximal time reduced. Therefore, $\tau$ values can indirectly reflect the characteristics of acoustic emission phenomenon parameters - the size of the ringing frequency.
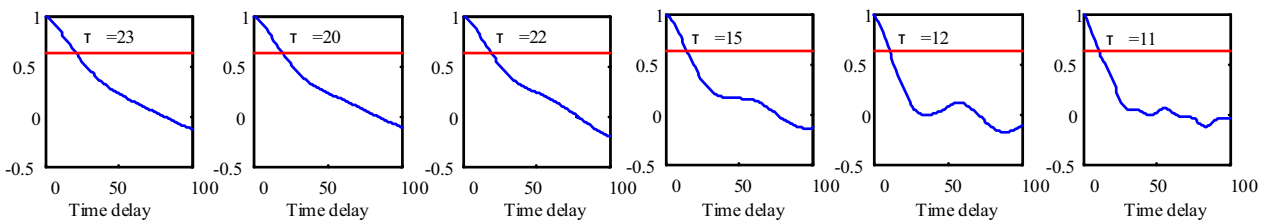

Figure 2. Autocorrelation method corresponding to different wear quantity signal delay time

(2)The CAO method for the embedding dimension

CAO solved minimum embedding dimension on the basis of FNN (False on his Neighbors). Table 1 is the delay time and embedding dimension based on the acoustic emission signal of different wear loss. The table shows that with the increase of wear and tear, the delay time and embedding dimension shows a trend of decrease.

Table 1. Corresponding to the calculation results of different wear loss

\begin{tabular}{ccccc}
\hline Wear & \multicolumn{2}{c}{ Delay time $\boldsymbol{\tau}$} & \multicolumn{2}{c}{ Embedding dimension $\mathbf{~}$} \\
\cline { 2 - 5 } loss/mm & Range & Mean & Range & Mean \\
\hline 0.10 & $20 \sim 25$ & 23 & $10 \sim 12$ & 10 \\
0.13 & $19 \sim 22$ & 20 & $9 \sim 13$ & 11 \\
0.16 & $20 \sim 23$ & 22 & $10 \sim 11$ & 11 \\
0.23 & $12 \sim 17$ & 15 & $9 \sim 12$ & 12 \\
0.27 & $10 \sim 13$ & 12 & $8 \sim 11$ & 8 \\
0.31 & $10 \sim 12$ & 11 & $8 \sim 10$ & 9 \\
\hline
\end{tabular}

\subsection{Attractor phase diagram.}

Each dimension of reconstructed phase space vector contains information of system evolution, two adjacent phase space vector can map the phase space trajectory, this is the attractor. Attractor has self-similar hierarchical structure, for a dissipative system, as the growth of the time, phase volume shrinks. Orbital folding, twisted and stretching, results unlimited nested self-similar geometric structure. It makes the trajectory of convergence to the attractor steadily. Figure 3 is attractor corresponding to the three different wear, respectively structured track structure from the $2 \mathrm{~d}$ and $3 \mathrm{~d}$. Attractor has obvious folding nested structure, and on the direction $\mathrm{y}=\mathrm{x}$, and tool wear acoustic emission signal has chaos characteristics.
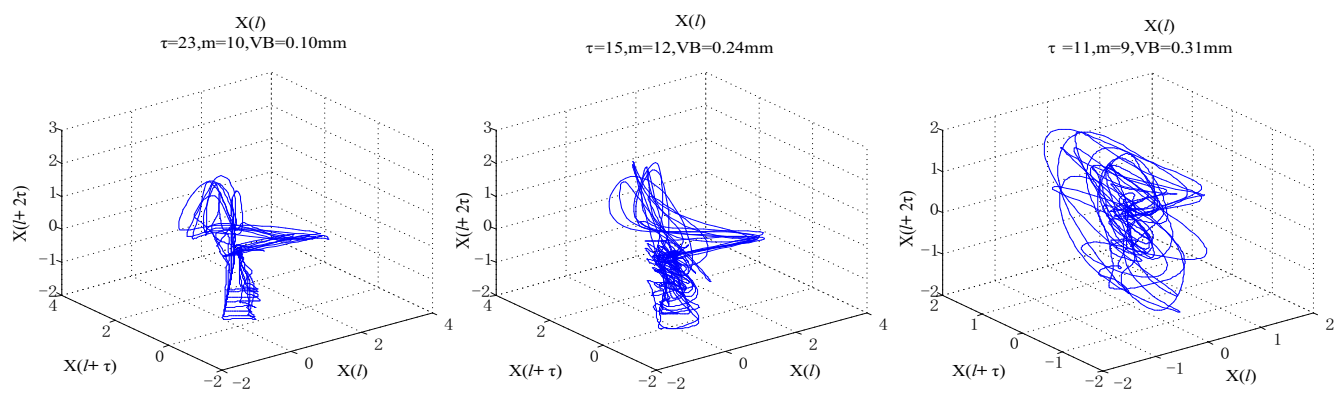

Figure 3. Attractor figures of different wear loss

\subsection{Correlation dimension.}


Correlation dimension is one of the types of extraction dimension, extracted from the chaos attractor in a series of points, and reflects the system complexity. This paper mainly calculated correlation dimension by GP algorithm. The main idea of the GP algorithm is to give two points $\mathrm{X}_{\mathrm{i}}, \mathrm{X}_{\mathrm{j}}$ in phase space, the distance between them is $r_{i j}$. The distance between the point and the point is closer, their correlation degree is higher. A time series in the $\mathrm{M}$ dimensional phase space generate $\mathrm{N}$ phase points, $\mathrm{X}_{1}, \mathrm{X}_{2}, \ldots, \mathrm{X}_{\mathrm{N}}$. Giving a positive number, the distance of points of logarithm is $r_{i j}<\varepsilon, C(\varepsilon)$ is the proportion of the total points of logarithm, there is:

$$
C(\varepsilon)=\frac{1}{N^{2}} \sum_{i, j=1, i \neq j}^{N} \theta\left(\varepsilon-\left|X_{i}-X_{j}\right|\right)
$$

$\theta(x)$ is the unit step function, correlation dimension is showed as follows:

$$
D=\lim _{\varepsilon \rightarrow 0} \frac{\ln C(\varepsilon)}{\ln \varepsilon}
$$

The type 5 shows that positive scale $\varepsilon$ determines the accuracy of the correlation dimension. In practical applications, there is no specific scale, by giving a $\mathrm{m}$, get a curve of $\ln \mathrm{C}(\varepsilon)$ and $\ln \varepsilon$, calculate a group of logarithmic curve corresponding to $\mathrm{m}$. Curve of linear scale-free in linear slope changes no longer and is saturated, and the straight slope is the correlation dimension. As it is shown in figure 4, the curve and the gradient of correlation dimensions $\operatorname{lnC}(\varepsilon)-\ln \varepsilon$ with the wear quantity is $0.24 \mathrm{~mm}$.
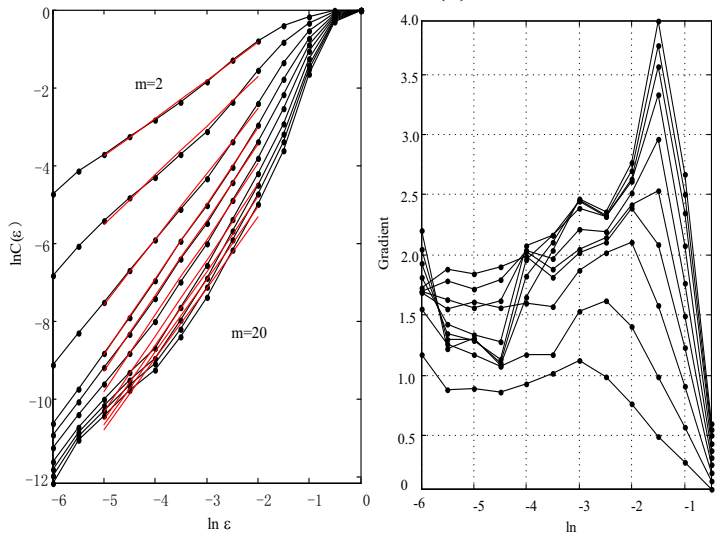

Figure 4. $\ln C(\varepsilon)$ and the gradient with $\ln \varepsilon$ diagram

Linear scale-free region straight slope is correlation dimension, and graphic correlation dimension is equal to 2.2052. The relations between wear loss and correlation dimension is shown in figure 5 .

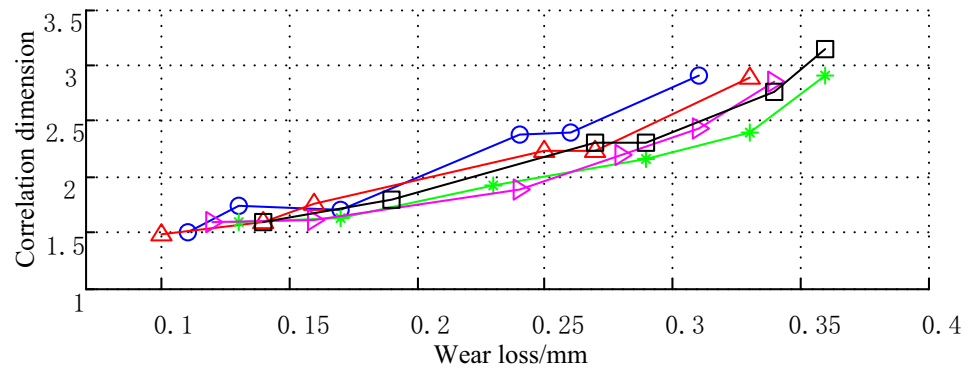

Figure 5. Different tool loss corresponds to the correlation dimension

As can be seen from the figure 5, as erosion rate increases, the increase of correlation dimension and nonlinear dynamic system is more complex, the adjacent sequence correlation decreases.

\subsection{Lyapunov index}


Lyapunov index is a kind of strange attractor nature of the measure and statistics, and maximum Lyapunov index reflects the system is in chaos. For one dimensional nonlinear dynamic system, Lyapunov index is expressed as:

$$
\lambda=\lim _{n \rightarrow 0} \frac{1}{n} \sum_{i=0}^{n-1} \ln \left|\frac{d f(x)}{d x}\right|_{x=x_{i}}
$$

If it is extended to the $\mathrm{n}$ power system, can get $\mathrm{n}$ Lyapunov index, $\lambda_{1} \geq \lambda_{2} \cdots \geq \lambda_{n}$. The smallest Lyapunov index determines the dynamic system orbit contraction speed, and maximum Lyapunov index reflects the speed of covering the track attractor. In this paper, calculate Lyapunov index according to the small data method [7].

Figure 6 shows the curve evolution of distance with the wear loss is $0.16 \mathrm{~mm}$, the basic orbit jth pair adjacent points, after i discrete time step. The red part of the figure is to use the least squares fitting to get maximum slope of the straight line segment, calculated maximum Lyapunov index is 0.2318 . It said that system has chaotic characteristics.

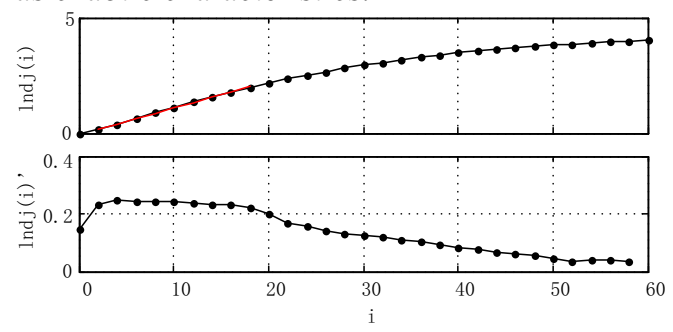

Figure 6. Linear area and the largest Lyapunov index curve

The sampling signal under different cutting condition Lyapunov index and correlation dimension showed that with the increase of wear, Lyapunov index and correlation dimension presented the same change trend, illustrates the enhancement of chaotic characteristic. It also reflects the state of tool wear.

\subsection{The Kolmogorov entropy.}

$\mathrm{K}$ entropy is an important characteristic of characterization of nonlinear system complexity, it says the average of the system information loss rate, and can represent chaos system. The mathematics is based on the definition of infinite subdivision, estimate the amount of information which is calculated by measure. Usually in order to simplify the calculation, according to thermodynamics entropy $\mathrm{S}$ to define Q order generalized entropy:

$$
K_{q}=\lim _{\tau \rightarrow 0} \lim _{r \rightarrow 0} \lim _{m \rightarrow \infty} \frac{1}{m \tau} \frac{1}{q-1} \ln \sum_{i=0}^{m} P^{q}\left(i_{0}, i_{1}, \cdots, i_{m}\right)
$$

When $\mathrm{q}=0$, it is the topological entropy, and when $\mathrm{q}=1$, $\mathrm{K}$ entropy.

GP algorithm gives the calculation method of K2. Correlation integral is shown in 10, combined with the correlation integral to get the following formula:

$$
K_{2}=\frac{1}{m \tau} \ln \frac{\varepsilon^{D}}{C(\varepsilon)}(\varepsilon \rightarrow 0)
$$

Figure 7 is K entropy's change curve with the embedding dimension. 


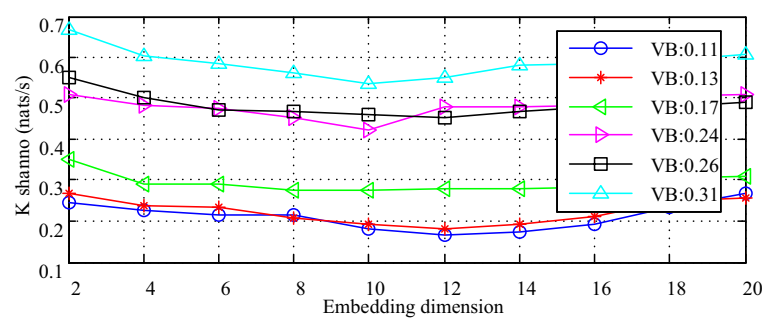

Figure 7. K entropy change with embedding dimension curve

As can be seen from the figure 7, the embedding dimension has less effect on the K entropy. With wear loss change, entropy $\mathrm{K}$ showed a trend of increase, wear was positively correlated with the chaos of the system.

\section{The application of SVM in the tool wear state recognition}

Support vector machine (SVM) is the latest in statistical learning theory and practical content, has a wide application in engineering field. Support vector machine classification, the main parameters are the kernel function is $t$, loss function, penalty coefficient $\mathrm{c}$ and kernel function parameter $\mathrm{g}$, they are need to be set up. Parameters' selection directly influences the final identification accuracy.

Choose features samples, they are under different conditions, different wear conditions. Take 60 samples into support vector machine (SVM) for training and recognition. Keep other parameters constant, change the kernel function type, get the recognition accuracy as shown in table 2 .

Table 2. Different kernel functions corresponding to the recognition accuracy under roughly selected parameters

\begin{tabular}{ccc}
\hline Kernel function type & Parameters' selection & Recognition accuracy \\
\hline Linear & '-c $1.2-\mathrm{g} 2.8-\mathrm{t}$ ' & $85.0000 \%$ \\
Polynomial & '-c $1.2-\mathrm{g} 2.8-\mathrm{t} 1$ ' & $96.6667 \%$ \\
RBF & '-c $1.2-\mathrm{g} 2.8-\mathrm{t} 2$ ' & $91.6667 \%$ \\
Sigmoid & '-c $1.2-\mathrm{g} 2.8-\mathrm{t} \mathrm{3}$ & $33.3333 \%$ \\
\hline
\end{tabular}

Table 2 is the recognition accuracy of different kernel functions, under the condition of roughing parameters. When the kernel function is polynomial kernel function, the recognition accuracy is higher. LIBSVM [8] can use Cross test Valid (Cross) to realize parameter optimization, referred to as $\mathrm{CV}$ method. The increase of the classification accuracy is more obvious after parameters optimized by $\mathrm{CV}$ method. Interactive inspection essence is to seek the optimal parameters under the condition of the best classification accuracy. Within a certain range, the two parameters is divided into a grid, finding the best parameters based on the given step value. The calculated best punishment coefficient $\mathrm{c}$ is 5.2780 , the kernel function parameter $\mathrm{g}$ is 48.5029 . As can be seen from table 3 , after the parameters were optimized, identification accuracy was obviously improved.

Table 3. Different kernel functions corresponding to the recognition accuracy after parameter optimization

\begin{tabular}{ccc}
\hline Kernel function type & Parameters' selection & Recognition accuracy \\
\hline Linear & 'c 5.2780-g 48.5029-t 0’ & $91.6667 \%$ \\
Polynomial & ‘-c 5.2780-g 48.5029-t 1 & $100 \%$ \\
RBF & ‘-c 5.2780-g 48.5029-t 2’ & $96.6667 \%$ \\
Sigmoid & ‘-c 5.2780-g 48.5029-t 3’ & $33.3333 \%$ \\
\hline
\end{tabular}




\section{Summary}

The chaotic characteristics of acoustic emission signals are determined by the tool wear condition, by reconstructing the phase space of the time sequence signals, the phase trajectory structure of the nonlinear dynamic system can be recovered. The complexity of the system can be characterized by chaos: correlation dimension, Lyapunov exponent, Kolmogorov entropy to measure. The constructed feature space of tool wear acoustic emission signal chaos features, combined with the SVM, can well realize classification of tool wear state. The selection of SVM kernel function has a great influence on the identification results, and the parameter optimization selection can improve the recognition accuracy of the model significantly.

\section{References}

1. Zhang Kai-feng, Yuan Hui-qun, Nie Peng. Tool wear condition monitoring based on generalized fractal dimensions[J]. Journal of Vibration and Shock, 2014, 33(1): 162-164.

2. Li Xiaoli. Fuzzy Pattern Recognition of the Wear of Metal Cutting Tools [J]. Journal of KunMing Institute of Technology, 1995, 20 (5):26-32.

3. Tae Jo KO, Dong Woo Cho, et al. Fuzzy Pattern Recognition for Tool Wear Monitoring in Diamond Turning[J].CIRP Annals-Manufacturing Technology, 1992, 41(1): 125-128.

4. Wafaa Rmili, Abdeljalil Ouahabi, et al. An automatic system based on vibratory analysis for cutting tool wear monitoring[J]. Measurement , 2016, 77(3): 117-123.

5. Li Gang-hu, Li Ya-an, JIA Xue-song. Better feature extraction and classification of chip-radiated noise with three chaos feature parameters[J].Journal of Northwestern Polytechnical University, 2006, 24(2): 170-173. (in Chinese with English abstract)

6. Volodymyr Lynnyk, Noboru Sakamoto, Sergej Čelikovský. Pseudo random number generator based on the generalized Lorenz chaotic system[J].IFAC-Papers OnLine, 2015, 48(18): 257-261.

7. Yang Yong-feng, Wu Min-juan, Gao Zhe, et al. Parameters selection for calculating largest lyapunov exponent from small datasets[J].Journal of Vibration, Measurement\& Diagnosis, 2012, 32(3): 371-374.

8. Wu Hao, Li Qun-zhan, Liu Wei. A new algorithm of wide-area backup protection based on PSO-LIBSVM[J]. Power System Protection and Control, 2013, 41(15): 49-58. 